\title{
Correction to: Advanced imaging in adult diffusely infiltrating low-grade gliomas
}

Nail Bulakbaşı ${ }^{1 *}$ and Yahya Paksoy ${ }^{2}$

\section{Correction to: Insights Imaging \\ https://doi.org/10.1186/s13244-019-0793-8}

The original article "Advanced imaging in adult diffusely infiltrating low-grade gliomas" contains errors in Table 1 in rows $k_{\text {trans }}$ and $V_{e}$; the correct version of Table 1 can be viewed in this Correction article. 
Table 1 Radiomic data for differential diagnosis of low-grade vs high-grade gliomas

\begin{tabular}{|c|c|c|c|}
\hline Parameters & Low-grade glioma & High-grade glioma & Cut of value Ref $^{\text {Range })^{\text {Ref }}}$ \\
\hline$r C B V_{\max }$ & Low & High & $1.76[10,19](0.94-3.34)[9-11,19-21]$ \\
\hline$r C B V_{M D}$ & Low & High & $1.44[22](1.08-1.81)[22]$ \\
\hline $\mathrm{nADC}$ min & High & Low & $1.07 \times 10^{-3} \mathrm{~mm}^{2} / \mathrm{s}[24](0.31-1.31)[23-28]$ \\
\hline Cho/Cr ratio & Low & High & $1.56[19](1.3-2.04)[29-31]$ \\
\hline$M K_{M D}$ & Low & High & $0.17[28](0.11-0.28)[28]$ \\
\hline$F A_{T C}$ & Low & High & $0.3[25](0.14-0.63)[25]$ \\
\hline$M D_{\min }$ & High & Low & $0.98 \mathrm{~mm}^{2} / \mathrm{s}[25](0.76-0.91)[25]$ \\
\hline$k_{\text {trans }}$ & Low & High & 1.18 [22] $(0.91-1.45)[22]$ \\
\hline$V_{e}$ & Low & High & $1.43[22](1.06-1.80)[22]$ \\
\hline ITTS grade & 1.2 & 2.6 & NA [9] \\
\hline APT signal (\%) & Low & High & $2.23 \%[15](1.53 \%-3.70 \%)[15]$ \\
\hline
\end{tabular}

$r C B V_{\text {max }}$ maximum relative cerebral blood volume, $r C B V_{M D}$ standardized mean difference of $r C B V_{\max }, n A D C_{\min }$ Normalized minimum apparent diffusion coefficient, $\mathrm{Cho} / \mathrm{Cr}$ Choline/Creatine, $M K_{M D}$ mean difference in mean kurtosis, $F A_{T C}$ Odds ratio of fractional anisotropy in the tumor core, $M D_{\min }$ Minimum mean diffusivity, $k_{\text {trans }}$ standardized mean difference of volume transfer coefficient, $V_{e}$ standardized mean difference of volume fraction of extravascular extracellular space, ITTS Intratumoral susceptibility score, APT Percent amide proton transfer signal

\section{Author details}

'Medical Faculty, University of Kyrenia, Sehit Yahya Bakır Street, Karakum, Mersin-10, Kyrenia, Turkish Republic of Northern Cyprus, Turkey. ${ }^{2}$ Selcuk University, Konya, Turkey.

Published online: 22 April 2020

\section{Reference}

1. Louis DN, Ohgaki H, Wiestler OD, Cavenee WK (2016) WHO classification oftumours of the central nervous system, revised 4th edition. IARC, Lyon

2. Sahm F, Reuss D, Koelsche C et al (2014) Farewell to oligoastrocytoma: insitu molecular genetics favor classification as either oligodendroglioma orastrocytoma. Acta Neuropathol 128(4):551-559. https://doi.org/10.1007/ s00401-014-1326-7

3. Bready D, Placantonakis DG (2019) Molecular pathogenesis of low-gradeglioma. Neurosurg Clin N Am 30(1):17-25. https://doi.org/10.1016/.jnec.2018.08.011

4. Duffau $\mathrm{H}$ (2016) Long-term outcomes after supratotal resection of diffuselowgrade gliomas: a consecutive series with 11-year follow-up. ActaNeurochir (Wien) 158(1):51-58. https://doi.org/10.1007/s00701-015-2621-3

5. National Comprehensive Cancer Network. Central nervous system cancers (Version 1.2019). https://www.nccn.org/professionals/physician_gls/pdf/cns. pdf. Accessed Mar 52019

6. Olson JJ, Kalkanis SN, Ryken TC (2015) Evidence-based clinical practiceparameter guidelines for the treatment of adults with diffuse lowgradeglioma: introduction and methods. J Neurooncol 125(3):449-456. https://doi.org/10.1007/s1 1060-015-1847-5.

7. Fouke SJ, Benzinger T, Gibson D, Ryken TC, Kalkanis SN, Olson JJ (2015) Therole of imaging in the management of adults with diffuse low-gradeglioma: a systematic review and evidence-based clinical practice guideline. JNeurooncol 125(3):457-479. https://doi.org/10.1007/s11060-015-1908-9

8. Caulo M, Panara V, Tortora D et al (2014) Data-driven grading of braingliomas: a multiparametric MR imaging study. Radiology 272(2):494503. https://doi.org/10.1148/radiol.14132040.

9. Li X, Zhu Y, Kang H et al. (2015) Glioma grading by microvascularpermeability parameters derived from dynamic contrast-enhanced MRI andintratumoral susceptibility signal on susceptibility weighted imaging. Cancerlmaging 15:4. https://doi.org/10.1186/s40644-015-0039-z

10. Delgado AF, Delgado AF (2017) Discrimination between glioma grades Iland III using dynamic susceptibility perfusion MRI: a meta-analysis. AJNR AmJ Neuroradiol 38(7):1348-1355. https://doi.org/10.3174/ajnr. A5218

11. Kong $L$, Chen $H$, Yang $Y$, Chen $L$ (2017) A meta-analysis of arterial spinlabelling perfusion values for the prediction of glioma grade. Clin Radiol72(3):255-261. https://doi.org/10.1016/j.crad.2016.10.016.

12. ACR Committee on Drugs and Contrast Media (2018) ACR manual oncontrast media (Version 10.3, 2018). https://www.acr.org/-/media/ACR/ Files/Clinical-Resources/Contrast_Media.pdf. Accessed 2018.
13. Choi C, Ganji SK, DeBerardinis RJ et al. (2012) 2-hydroxyglutarate detectionby magnetic resonance spectroscopy in IDH-mutated patients with gliomas. Nat Med 18(4):624-629. https:/doi.org/10.1038/nm.2682.

14. Tietze A, Choi C, Mickey B et al. (2018) Noninvasive assessment of isocitratedehydrogenase mutation status in cerebral gliomas by magnetic resonancespectroscopy in a clinical setting. J Neurosurg 128(2):391-398. https://doi.org/10.3171/2016.10.JNS161793

15. Suh CH, Park JE, Jung SC, Choi CG, Kim SJ, Kim HS (2019) Amide protontransfer-weighted MRI in distinguishing high- and low-grade gliomas: asystematic review and meta-analysis. Neuroradiology 61(5):525-534. https://doi.org/10.1007/s00234-018-02152-2

16. Park JE, Kim HS, Park KJ, Choi CG, Kim SJ (2015) Histogram analysis of amideproton transfer imaging to identify contrast-enhancing low-grade braintumor that mimics high-grade tumor: increased accuracy of MR perfusion.Radiology 277(1):151-161. https://doi.org/10.1148/radiol.2015142347.

17. Biller A, Badde S, Nagel A et al. (2016) Improved brain tumor classification bysodium MR imaging: prediction of IDH mutation status and tumor progression. AJNR Am J Neuroradiol 37(1):66-73. https://doi.org/10.3174/ajnr. A4493

18. Pepin KM, McGee KP, Arani A et al. (2018) MR elastography analysis ofglioma stiffness and IDH1-mutation status. AJNR Am J Neuroradiol 39(1): 31-36. https://doi.org/10.3174/ajnr. A5415

19. Law M, Yang S, Wang H et al. (2003) Glioma grading: sensitivity, specificity, and predictive values of perfusion MR imaging and proton MRspectroscopic imaging compared with conventional MR imaging. AJNR AmJ Neuroradiol 24(10):1989-1998

20. Bulakbasi N, Kocaoglu M, Farzaliyev A, Tayfun C, Ucoz T, Somuncu I (2005) Assessment of diagnostic accuracy of perfusion MR imaging in primary andmetastatic solitary malignant brain tumors. AJNR Am J Neuroradiol 26(9):2187-2199

21. Anzalone N, Castellano A, Cadioli M et al. (2018) Brain gliomas: multicenterstandardized assessment of dynamic contrast-enhanced and dynamicsusceptibility contrast MR images. Radiology 287(3):933-943. https://doi.org/10.1148/radiol.2017170362

22. Liang J, Liu D, Gao P et al. (2018) Diagnostic values of DCE-MRI and DSCMRIfor differentiation between high-grade and low-grade gliomas: acomprehensive meta-analysis. Acad Radiol 25(3):338-348. https://doi.org/ 10.1016/j.acra.2017.10.001

23. Bulakbasi N, Guvenc I, Onguru O, Erdogan E, Tayfun C, Ucoz T (2004) Theadded value of the apparent diffusion coefficient calculation to magneticresonance imaging in the differentiation and grading of malignant braintumors. J Comput Assist Tomogr 28(6):735-46

24. Server A, Kulle B, Gadmar ØB, Josefsen R, Kumar T, Nakstad PH (2011) Measurements of diagnostic examination performance using quantitativeapparent diffusion coefficient and proton MR spectroscopic imaging in thepreoperative evaluation of tumor grade in cerebral gliomas. Eur J Radiol 80(2):462-70. https://doi.org/10.1016/j.jrad.2010.07.017 
25. Miloushev VZ, Chow DS, Filippi CG (2015) Meta-analysis of diffusion metricsfor the prediction of tumor grade in gliomas. AJNR Am J Neuroradio 36(2):302-8. https://doi.org/10.3174/ajnr.A4097

26. Zhang L, Min Z, Tang M, Chen S, Lei X, Zhang X (2017) The utility ofdiffusion MRI with quantitative ADC measurements for differentiating high-grade from low-grade cerebral gliomas: evidence from a meta-analysis. JNeurol Sci 373:9-15. https://doi.org/10.1016/j.jns.2016.12.008Erratum in: JNeurol Sci. 2017;375:103-6

27. Liang R, Wang X, Li M et al. (2014) Potential role of fractionalanisotropy derived from diffusion tensor imaging in differentiatinghigh-grade gliomas from low-grade gliomas: a meta-analysis. Int J ClinExp Med 7(10):3647-53

28. Falk Delgado A, Nilsson M, van Westen D, Falk Delgado A (2018) Gliomagrade discrimination with MR diffusion kurtosis imaging: a metaanalysis ofdiagnostic accuracy. Radiology 287(1):119-27. https://doi.org/10. 1148/radiol.2017171315

29. Castillo M, Smith JK, Kwock L (2000) Correlation of myo-inositol levels andgrading of cerebral astrocytomas. AJNR Am J Neuroradiol 21(9):1645-49.

30. Wang Q, Zhang H, Zhang J et al. (2016) The diagnostic performance ofmagnetic resonance spectroscopy in differentiating high-from lowgradegliomas: a systematic review and meta-analysis. Eur Radiol 26(8):267084. https://doi.org/10.1007/s00330-015-4046-z

31. Usinskiene J, Ulyte A, Bjørnerud A et al. (2016) Optimal differentiation ofhigh- and low-grade glioma and metastasis: a meta-analysis of perfusion, diffusion, and spectroscopy metrics. Neuroradiology 58(4):339-50. https:// doi.org/10.1007/s00234-016-1642-9

32. Hilario A, Sepulveda JM, Perez-Nuñez A et al. (2014) A prognostic modelbased on preoperative MRI predicts overall survival in patients with diffusegliomas. AJNR Am J Neuroradiol 35(6):1096-1102. https:/doi.org/10.3174/ajnr.A3837

33. Cuccarini V, Erbetta A, Farinotti M et al. (2016) Advanced MRI maycomplement histological diagnosis of lower grade gliomas and help inpredicting survival. J Neurooncol 126(2):279-88

34. Villanueva-Meyer JE, Wood MD, Choi BS et al. (2018) MRI features and IDHmutational status of grade II diffuse gliomas: impact on diagnosis andprognosis. AJR Am J Roentgenol 210(3):621-28. https://doi.org/10.2214/ AJR.17.18457

35. Law M, Oh S, Babb JS et al. (2006) Low-grade gliomas: dynamicsusceptibility-weighted contrast-enhanced perfusion MR imagingpredictionof patient clinical response. Radiology. 238(2):658-67

36. Nguyen TB, Cron GO, Mercier JF et al. (2015) Preoperative prognostic valueof dynamic contrast-enhanced MRI-derived contrast transfer coefficient andplasma volume in patients with cerebral gliomas. AJNR Am J Neuroradiol36(1):63-69. https://doi.org/10.3174/ajnr.A4006

37. Hlaihel C, Guilloton L, Guyotat J, Streichenberger N, Honnorat J, Cotton F (2010) Predictive value of multimodality MRI using conventional, perfusion, and spectroscopy MR in anaplastic transformation of lowgradeoligodendrogliomas. J Neurooncol 97(1):73-80. https://doi.org/10. 1007/s11060-009-9991-4

38. Law M, Young RJ, Babb JS et al. (2008) Gliomas: predicting time toprogression or survival with cerebral blood volume measurements atdynamic susceptibility-weighted contrast-enhanced perfusion MR imaging.Radiology 247(2):490-98. https://doi.org/10.1148/radiol.2472070898

39. Danchaivijitr N, Waldman AD, Tozer DJ et al. (2008) Low-grade gliomas: dochanges in rCBV measurements at longitudinal perfusion-weighted MRimaging predict malignant transformation? Radiology 247(1):170-78. https://doi.org/10.1148/radiol.2471062089

40. Back M, Jayamanne DT, Brazier D et al. (2019) Influence of molecularclassification in anaplastic glioma for determining outcome and futureapproach to management. J Med Imaging Radiat Oncol 63(2):272-80. https://doi.org/10.1111/1754-9485.12850

41. Shirahata M, Ono T, Stichel D et al. (2018) Novel, improved gradingsystem(s) for IDH-mutant astrocytic gliomas. Acta Neuropathol 136(1):153-66. https:// doi.org/10.1007/s00401-018-1849-4

42. Patel SH, Poisson LM, Brat DJ et al. (2017) T2-FLAIR mismatch, an imagingbiomarker for IDH and $1 \mathrm{p} / 19 \mathrm{q}$ status in lower-grade gliomas: a TCGA/TCIA project. Clin Cancer Res 23(20):6078-85. https://doi.org/10.1158/ 1078-0432.CCR-17-0560

43. Ren Y, Zhang X, Rui W et al. (2019) Noninvasive prediction of IDH1 mutationand ATRX expression loss in low-grade gliomas using multiparametric MRradiomic features. J Magn Reson Imaging 49(3):808-17. https://doi.org/10.1002/jmri.26240
44. Hasselblatt M, Jaber M, Reuss D et al. (2018) Diffuse astrocytoma, IDHwildtype: a dissolving diagnosis. J Neuropathol Exp Neurol 77(6):422-25. https://doi.org/10.1093/jnen/nly012

45. BratDJ, AldapeK, ColmanH, etal. (2018) cIMPACT-NOWupdate3: recommended diagnostic criteria for "diffuse astrocytic glioma, IDHwildtype, with molecular features of glioblastoma, WHO grade IV. Acta Neuropathol 136(5):805-10. https://doi.org/10.1007/s00401-018-1913-0

46. Wu CC, Jain R, Radmanesh A et al. (2018) Predicting genotype and survivalin glioma using standard clinical MR imaging apparent diffusion coefficientimages: a pilot study from the cancer genome atlas. AJNR Am J Neuroradiol 39(10):1814-20. https://doi.org/10.3174/ajnr.A5794

47. Smits M, van den Bent MJ (2017) Imaging correlates of adult gliomagenotypes. Radiology 284(2):316-31. https://doi.org/10.1148/radiol.2017151930

48. Kickingereder P, Sahm F, Radbruch A et al. (2015) IDH mutation status isassociated with a distinct hypoxia/angiogenesis transcriptome signaturewhich is non-invasively predictable with rCBV imaging in human glioma. SciRep 5:16238. https://doi.org/10.1038/srep16238

49. Leu K, Ott GA, Lai A et al. (2017) Perfusion and diffusion MRI signatures inhistologic and genetic subtypes of WHO grade II-III diffuse gliomas. JNeurooncol 134(1):177-88. https://doi.org/10.1007/s11060-017-2506-9

50. Stadlbauer A, Zimmermann M, Kitzwögerer M et al. (2017) MR imagingderived oxygen metabolism and neovascularization characterization forgrading and IDH gene mutation detection of gliomas. Radiology 283(3): 799-809. https://doi.org/10.1148/radiol.2016161422

51. Bian W, Khayal IS, Lupo JM et al. (2009) Multiparametric characterization ofgrade 2 glioma subtypes using magnetic resonance spectroscopic, perfusion, and diffusion imaging. Transl Oncol 2(4):271-280

52. Lin Y, Xing Z, She D et al. (2017) IDH mutant and 1p/19q codeletedoligodendrogliomas: tumor grade stratification using diffusion-, susceptibility-, and perfusion-weighted MRI. Neuroradiology 59(6):555-62. https://doi.org/10.1007/s00234-017-1839-6

53. Yoon HJ, Ahn KJ, Lee $S$ et al. (2017) Differential diagnosis of oligodendroglialand astrocytic tumors using imaging results: the added value of perfusionMR imaging. Neuroradiology 59(7):665-75. https://doi.org/10.1007/s00234-017-1851-x

54. Jenkinson MD, Smith TS, Joyce KA et al. (2006) Cerebral blood volume, genotype and chemosensitivity in oligodendroglial tumours.Neuroradiology 48(10):703-13.

55. Chawla S, Krejza J, Vossough A et al. (2013) Differentiation betweenoligodendroglioma genotypes using dynamic susceptibility contrastperfusion-weighted imaging and proton MR spectroscopy. AJNR Am JNeuroradiol 34(8):1542-49. https://doi.org/10.3174/ajnr.A3384

56. Emblem KE, Scheie D, Due-Tonnessen $P$ et al. (2008) Histogram analysis ofMR imaging-derived cerebral blood volume maps: combined gliomagrading and identification of low-grade oligodendroglial subtypes. AJNRAm J Neuroradiol 29(9):1664-70. https://doi.org/10.3174/ajnr.A1182

57. Emblem KE, Nedregaard B, Nome T et al. (2008) Glioma grading by usinghistogram analysis of blood volume heterogeneity from MRderivedcerebral blood volume maps. Radiology 247(3):808-17. https://doi. org/10.1148/radiol.2473070571

58. Dietrich O, Reiser MF, Schoenberg SO (2008) Artifacts in 3T MRI: physicalbackground and reduction strategies. Eur J Radiol 65(1):29-35

59. Vargas MI, Delavelle J, Kohler R, Becker CD, Lovblad K (2009) Brain and spineMRI artifacts at 3Tesla. J Neuroradiol 36(2):74-81. https://doi.org/10. 1016/j.neurad.2008.08.001

60. Kang Y, Choi SH, Kim YJ (2011) Gliomas: histogram analysis of apparentdiffusion coefficient maps with standard- or high-b-value diffusionweighted MR imaging--correlation with tumor grade. Radiology 261(3):88290. https://doi.org/10.1148/radiol.11110686

61. Zhou M, Scott J, Chaudhury B et al. (2018) Radiomics in brain tumor: imageassessment, quantitative feature descriptors, and machinelearningapproaches. AJNR Am J Neuroradiol 39(2):208-16. https://doi.org/10. 3174/ajnr.A5391

62. Zhang $X$, Yan LF, Hu YC et al. (2017) Optimizing a machine learning basedglioma grading system using multi-parametric MRI histogram and texturefeatures. Oncotarget 8(29):47816-830. https://doi.org/10.18632/ oncotarget.18001

63. Lee MH, Kim J, Kim ST et al. (2019) Prediction of IDH1 mutation in GBMusing machine learning technique based on quantitative radiomic data.World Neurosurg 125:e688-e696. https://doi.org/10.1016/j.wneu.2019.01.157 